\title{
Pre-Patterns for Designing Embodied Interactions in Handheld Augmented Reality Games
}

\author{
Yan Xu*, Evan Barba*, Iulian Radu*, Maribeth Gandy*, Richard \\ Shemaka*, Brian Schrank*, Blair Maclntyre* \\ Augmented Environments Lab, Georgia Institute of Technology
}

\author{
Tony Tseng ${ }^{\dagger}$ \\ Savannah College of Art and Design
}

\begin{abstract}
The game industry and related research communities have shown a surge of interest in reality-based interfaces that create "embodied" game play experiences. Handheld AR (HAR) is a reality-based interface that renders digital objects onto a player's perception of the physical world. HAR creates a hybrid space in which players can leverage their existing physical and social skills to interact with the game system and with each other. Although HAR has received some attention in the world of handheld gaming, there is little research that summarizes and communicates design principles and implications across multiple examples. In this paper, we analyze and generate design lessons from dozens of HAR games, drawn from academic and commercial AR games, and also our years of experience designing and teaching HAR game design. We summarize our experience in this new field into a set of design "pre-patterns" as a means of formalizing significant design lessons derived from these existing practices into repeatable principles and solutions. We contribute to both the game and interaction design communities with pre-patterns that support embodied game play.
\end{abstract}

KEYWORDS: Handheld augmented reality interface, design patterns, game design, game interface.

INDEX TERMS: Index Terms: H.5.1 [INFORMATION INTERFACES AND PRESENTATION (e.g., HCI)]: Multimedia Information Systems - Artificial, augmented, and virtual realities; K.8.0 [PERSONAL COMPUTING]: General- Games.

\section{INTRODUCTION}

The current trend in the game industry, exemplified by the Nintendo Wii [1] and Microsoft Kinect [2], is toward game interfaces that leverage players' embodied knowledge and skills to create experiences that are natural and intuitive, and often more social than previous console systems allowed. Handheld Augmented Reality (HAR) interfaces, where the computergenerated content is registered with the player's view of the physical world in real time, is one type of such "reality-based interfaces" [3], which draw strength from users' pre-existing knowledge and skills [4]. Although HAR games do not currently have a large share of the market, Augmented Reality (AR) gaming is beginning to gain popularity [5]. For example, Nintendo's $3 D S$ [6] comes pre-packaged with a number of $\mathrm{AR}$ games and developers are beginning to create $\mathrm{AR}$ games for mainstream

* Email: \{yan.xu, ebarba3, iulian.radu, maribeth.gandy, rshemaka, bschrank, blair\}@gatech.edu

†Email: ttseng@scad.edu

LEAVE 0.5 INCH SPACE AT BOTTOM OF LEFT COLUMN ON FIRST PAGE FOR COPYRIGHT BLOCK smart phones. Unfortunately, designing games for a new technology can be challenging until the design community has more experience with the specific affordances and constraints of the interfaces. The goal of this paper is to share the experience we have gained over the past four years designing and evaluating HAR games [7-9], observing and guiding novice game designers who worked with HAR for the first time in AR game studio ${ }^{1}$ [10], and analysing dozens of examples from both industry and academia.

As new designers and researchers approach HAR technology, they are asking themselves similar questions. What kind of experience can HAR support and create for the player, aside from the shear "novelty" of the interface? What are the constraints and complexities that this technology adds? How can we transform embodied interaction with a HAR interface into meaningful embodied game play? Both AR research and game design communities have something to contribute to the discussion. AR researchers have investigated the affordances and constraints of AR interfaces [11,12], and empirically examined AR systems in a number of contexts, including collaborative work, instructor support, learning, and games [7,13-18]. Game designers are familiar with game elements and conventions that are critical for enjoyable game experiences. We hope to bridge this discussion between the two communities by presenting our results as a collection of design pre-patterns for HAR games.

Design pattern research is a tool to capture and communicate repeated solutions for recurring problems in the field [19-21]. Although it was first created in the context of the mature field of architecture, recent research has suggested the idea that sharing design knowledge might also be appropriate for less mature design domains, such as ubiquitous computing and home technology $[22,23]$. Recognizing that HAR game design is a nascent, fast-growing domain, we adopt the term "pre-patterns" in our work, which has been used to identify patterns that focus on summarizing ongoing practice to inspire new design.

In this paper, we present nine design pre-patterns that leverage four kinds of embodied human skills [4]. Different from software engineering design patterns, where the "problems" are usually clear and task-oriented [20], game designers face the challenge of experience design, which is essentially generated by players and over which a designer has only indirect control [24]. We focus on this experiential aspect of game design $[24,25]$ by adopting the lens of embodied interaction [26,27] to identify the underlying humanistic factors that these pre-patterns rely on.

These pre-patterns contribute to bridging the gap between interaction and game design. They give designers some basic building blocks for their own games, help them avoid common pitfalls, and understand how to craft the game experiences they want using this new technology. This work serves as a starting point for a complete pattern language of $\mathrm{AR}$ experiences that includes a broad spectrum of AR technologies and experiences.

${ }^{1}$ Qualcomm Augmented Reality Game Studio, www.argamestudio.org 


\section{EMBODIED INTERACTION}

The term "embodiment" can mean several related things, all of which are applicable to the design of AR experiences. For cognitive scientists, the term originates in the work of Lakoff and Johnson [26], which serves as the basis for the "embodied" view of cognition. Embodied cognition posits that our understanding and interpretation of the world around us is rooted in our, often unconscious, experience of our bodies. Through bodily interactions with the physical world, we develop "image schemas" that encode the structure and relationships learned in these encounters. These schemas are then adapted and applied to future experiences through the cognitive process of "metaphorical mapping." For HAR games, this concept provides a model of the cognitive processes of players, who often approach unfamiliar AR experiences from the standpoint of previous experiences with video games although mappings from other gaming experiences, such as board games, might be more appropriate. For AR experiences in general, players are likely to leverage previous experiences of navigating and manipulating the physical world. Designers must know how to determine what metaphors players are using, as well as how to leverage these associations so that users can engage with the game play correctly.

A related notion of "embodied interaction" has been offered by Dourish [27], and has become popular in HCI and ubiquitous computing research. According to this view, embodiment calls for computational resources to be physically embedded in the world, so that ordinary interactions with physical objects can have effects in the virtual world, thus creating a tight coupling between the physical and digital domains. This notion has obvious relation to the embodied cognition view, in that it focuses on unconscious or intuitive interactions in space, however, where embodied cognition tries to understand the role these play in the conceptual framework of the human mind, the embodied view of interaction emphasizes their role as input and output mechanisms in a humanmachine system. This too is a useful paradigm for HAR research, as the ability to project virtual spaces onto the physical world creates numerous opportunities to interact with physical objects. Understanding how to communicate the virtual affordances of physical objects to players, or to create physical objects with affordances specific to the hybrid reality of the game itself, are important considerations for HAR game designers and one of the issues addressed in our design patterns.

Embodied interactions rely on a person's existing embodied skills in everyday life (naïve physics, body awareness and skills, environmental awareness and skills, social awareness and skills) [4] and create experiences that are more intuitive, have more freedom of control, and more emotional and social engagement. We adopt the concept as a lens when we analyze design prepatterns and try to understand how the HAR interface brings embodied interaction to game play.

\section{ReLated Work}

\subsection{Pattern Languages}

"Each pattern describes a problem which occurs over and over again in our environment, and then describes the core of the solution to that problem in such a way that you can use this solution a million times over, without ever doing it the same way twice." - Christopher Alexander et al. [19]

In the past forty years, design patterns have spread from the field of architecture, where they were first observed, to numerous other fields such as, software engineering [4], human-computer interaction [21-23,28-30], and game design [25,31,32]. Researchers have previously adopted design patterns for the software engineering of AR systems [33].
The purpose of a design pattern is to summarize existing practices that are successful and significant, encapsulate this knowledge into a common format, and facilitate continuous communication for the community [21]. Design patterns are not just made of these individual patterns, but the hierarchy between these patterns, which is called pattern language.

Design patterns have been more widely adopted in the game design community in the past five years. Björk and Holopainen summarized a large set of game design patterns based on the game play experiences they support [25]. Seif et al. summarized design patterns for cooperative games through video analysis, including camera setting, interacting with the game objects, shared puzzles, shared character, limited resources, etc. [31] Along similar lines, we use design patterns as a research tool to summarize and communicate the existing practices found in HAR games in certain formats. However, we recognize that HAR games are still in the early stages of development and we therefore use the term "pre-patterns", following the examples from other nascent fields [22][23].

\subsection{Reality-based Interfaces in Games}

The discussion linking game interfaces with game play experiences has been ongoing for the past three decades [34-36]. There are many commercial examples where these interfaces are exploited in games for their novelty effect (and sales attraction). However, the affordances of these interfaces are often not actively integrated to the core game play [36].

Researchers have conducted empirical studies to understand how reality-based interfaces change game experiences. Lindley et al. found the amount of literal and non-literal communication increased when physical interfaces are used for co-located players [37]. Additionally, Mueller et al.'s research showed that exertion interface-based games can enhance social bonding and enjoyment for the remote players [38].

Game researchers and designers try to understand the role of interfaces in creating player experiences. Interfaces are part of the game, through which a player perceives, controls, and changes game states [24]. Game interfaces include both physical and digital components. For example, the joystick is the physical input, and it is mapped to the control of a digital character [24] Game interfaces support and integrate with other elements of the game, such as player actions, character design, space design and social play etc. [24,39] Media researchers also examined a unique type of games in which mastering the interface is the challenging part and subsequently becomes the core of the game play [40]. The tight relationship between interface and play experience is examined in our design pre-patterns.

\subsection{AR interface and HAR Games}

Augmented reality (AR) is one type of reality-based interface. AR interfaces supplement the real world with virtual (computergenerated) objects that appear to coexist in the same space as the real world [3]. With AR interfaces, users can contextualize digital information in the physical world [12], leveraging real-world physics, as well as their bodily and environmental awareness in the interactions. Moreover, for multiple users, AR enables them to view a hybrid space from their own perspective, while leveraging their verbal and nonverbal cues for face-to-face or remote collaboration $[11,41]$. Games are one of the biggest domains that AR interfaces have been applied to. A survey of AR games can be found in [42], where the authors mapped the games long the dimensions of time, technology and genre.

Currently the most commercialized AR games are on common hardware platforms, such as mirror image based AR with game consoles, and smart phones that have back cameras. While The 
Eye of Judgment [43] was the first commercially available augmented reality game, there have been a number of research projects devoted to handheld or tabletop AR games, such as the Invisible Train [44], AR Tennis [45], Cows vs. Aliens [46], and others.

With hardware improvements on handheld devices, the majority of smart phones become platforms for HAR games. Commercial games like InviZimals [47], EyePet (PSP version) [48], and Nintendo 3DS AR showcase games [6] have been created by large companies and launched in the past two years. With the available AR toolkits on smart phone platforms, such as the Qualcomm AR SDK for Android and iOS, independent game designers have also become interested in integrating HAR interfaces into their games. In our work, we draw from dozens of examples found in both commercial and independent games.

\section{METHODS}

\subsection{Choice of Design Pattern Method}

There are a number of options for codifying and communicating accumulated design knowledge and practice. For large sample sets, taxonomies are often preferred for grouping ideas according to similar features, and for organizing concepts hierarchically. However, because HAR games are still in their infancy, we do not yet have the breadth of examples and recurring design concepts required to create a useful taxonomy.

A second possible approach is the generation of "design heuristics" [34,49,50], which summarize knowledge about "good design" into rules and principles that serve as a checklist for evaluation. However, heuristics typically offer only a loose collection of "rules of thumb," lack the formal organization and categorization achieved by taxonomies, and do not suggest solutions when guidelines are not met, or causes when instances violate any particular heuristic. Still, these suggestions for design can be important, particularly for novice designers.

We believe that design patterns and pre-patterns are a good middle ground between taxonomies and heuristics. We acknowledge that the pre-patterns we present in this paper are only the beginning of an evolutionary process, and that all the connections and interconnections have not yet been clarified. We believe that as this field matures, accumulated knowledge will demonstrate patterns that have more well-understood relationships with each other, and that these will form the basis for a true pattern language of HAR design.

\subsection{Process of Generating HAR Game Pre-patterns}

We generated design pre-patterns through an iterative process that includes individual researchers' analysis of HAR games and group discussions about the significance and relevance of prepatterns. We started with one researcher extracting design features from all the games that we had access to. Then we categorized similar features together to form an initial set of pre-patterns, which were discussed among a group of researchers and designers who have at least one-year of experience designing and evaluating HAR games. In the past two months, we had eight discussion sessions that involve three to six participants each. We present nine of the most significant pre-patterns, grounded in our analysis of embodied interaction and in existing HAR games.

\subsection{Format of the Pre-pattern}

The format of design patterns varies by different researchers and domains. The commonality between them is to describe the problem (or the experience that can be achieved through the pattern) and solution in detail. We compared styles in other researchers' work in related domains, such as game design [25] and interaction design [28], and settled on a variation of Björk and Holopainen's game design pattern template. We simplify this template into the sections listed below because the relations between pre-patterns is unclear now, and will presumably emerge with future research.

- Title: a short phase that is memorable.

- Definition: A brief summary of what experiences the prepattern supports and how they are achieved.

- Description: A detailed explanation of how and why the prepattern works, what aspects of game design it is based on.

- Examples: Illustrate the meaning of the pre-pattern through examples.

- Using the pre-patterns: A pre-pattern can be used to guide practice. This part is dedicated to reveal the challenges and context of applying the pre-patterns.

\section{Design Pre-Patterns}

In this section, we present nine design patterns that leverage different embodied skills, the following table summarizes:

Table 1. Summary of the nine design pre-patterns

\begin{tabular}{|c|c|c|}
\hline Title & Meaning & $\begin{array}{l}\text { Embodied } \\
\text { Skills }\end{array}$ \\
\hline $\begin{array}{c}\text { Device } \\
\text { Metaphors }\end{array}$ & $\begin{array}{l}\text { Using metaphor to suggest available } \\
\text { player actions }\end{array}$ & $\begin{array}{c}\text { Body A\&S } \\
\text { Naïve physics }\end{array}$ \\
\hline $\begin{array}{l}\text { Control } \\
\text { Mapping }\end{array}$ & $\begin{array}{l}\text { Intuitive mapping between physical } \\
\text { and digital objects }\end{array}$ & $\begin{array}{c}\text { Body A\&S } \\
\text { Naïve physics }\end{array}$ \\
\hline $\begin{array}{l}\text { Seamful } \\
\text { Design }\end{array}$ & $\begin{array}{l}\text { Making sense of and integrating the } \\
\text { technological seams through game } \\
\text { design }\end{array}$ & Body A\&S \\
\hline $\begin{array}{c}\text { World } \\
\text { Consistency }\end{array}$ & $\begin{array}{l}\text { Whether the laws and rules in } \\
\text { physical world hold in digital world }\end{array}$ & $\begin{array}{c}\text { Naïve physics } \\
\text { Environmental } \\
\text { A\&S }\end{array}$ \\
\hline Landmarks & $\begin{array}{l}\text { Reinforcing the connection between } \\
\text { digital-physical space through } \\
\text { landmarks }\end{array}$ & $\begin{array}{l}\text { Environmental } \\
\text { A\&S }\end{array}$ \\
\hline $\begin{array}{l}\text { Personal } \\
\text { Presence }\end{array}$ & $\begin{array}{l}\text { The way that a player is represented } \\
\text { in the game decides how much they } \\
\text { feel like living in the digital game } \\
\text { world }\end{array}$ & $\begin{array}{l}\text { Environmental } \\
\qquad \text { A\&S } \\
\text { Naïve physics }\end{array}$ \\
\hline $\begin{array}{l}\text { Living } \\
\text { Creatures }\end{array}$ & $\begin{array}{l}\text { Game characters that are responsive } \\
\text { to physical, social events that mimic } \\
\text { behaviours of living beings }\end{array}$ & $\begin{array}{l}\text { Social A\&S } \\
\text { Body A\&S }\end{array}$ \\
\hline $\begin{array}{c}\text { Body } \\
\text { constraints }\end{array}$ & $\begin{array}{l}\text { Movement of one's body position } \\
\text { constrains another player's action }\end{array}$ & $\begin{array}{l}\text { Body A\&S } \\
\text { Social A\&S }\end{array}$ \\
\hline $\begin{array}{l}\text { Hidden } \\
\text { information }\end{array}$ & $\begin{array}{l}\text { The information that can be hidden } \\
\text { and partially revealed can foster } \\
\text { emergent social play }\end{array}$ & $\begin{array}{l}\text { Social A\&S } \\
\text { Body A\&S }\end{array}$ \\
\hline
\end{tabular}

*(A\&S is short for Awareness \& Skills)

Before introducing the patterns, we briefly summarize a few small design lessons into a list of dos and don'ts, which are selfexplanatory. Some of them are based on the current state of the art in HAR technology.

- Support one-hand interaction

- Consider the natural viewing angle

- Make sure at least one tracking surface is in view

- Do not tire players out physically

- Do not encourage fast actions 


\subsection{Device Metaphors}

Definition: Device metaphors guide players' actions by suggesting that the handheld device functions as a familiar object from everyday life.

Description: Due to the fact that $A R$ games are still new to many players, the conventions of this medium have not been well established. One effective way to solve this problem is to leverage the power of metaphor. For example, graphical user interfaces made a leap from the command line interface by adopting the metaphors like "desktop", "folder" and "trash bin", which are physical objects that imply the functions of digital objects. For reality-based interfaces, the usage of can suggest possible actions [52]. For example, the game of Wii Sports is easy to learn because the controller can take the metaphorical role of a racquet or bat, thus cognitively guiding a player's actions to those that are appropriate to those objects, such as swinging and tilting.

The physical actions for controlling a HAR interface can be implied through device metaphors. The HAR interface can have similarities to many kinds of lenses, such as cameras, magnifiers, microscopes, and $x$-ray machines. Players can naturally transfer what they know about using these devices to possible actions with the HAR interface. A game should reinforce the metaphor through appropriate visual, auditory, and haptic cues, ensuring that players do not make false inferences about the metaphor.

Examples: In the game Bug Juice, the handheld device is metaphorically linked to a magnifying glass. In this game, a player needs to stay at a certain distance to burn ants with the device, which might resonate with memories of focusing sunlight via a magnifying glass. Another benefit of this metaphor is that it guides the player to stay at a specific distance from the target that is optimal for the AR tracking software. The metaphor makes sense of the actions in the game and helps players learn these actions easier. This is also an example for another pattern, "design for the seams", which will be introduced later.

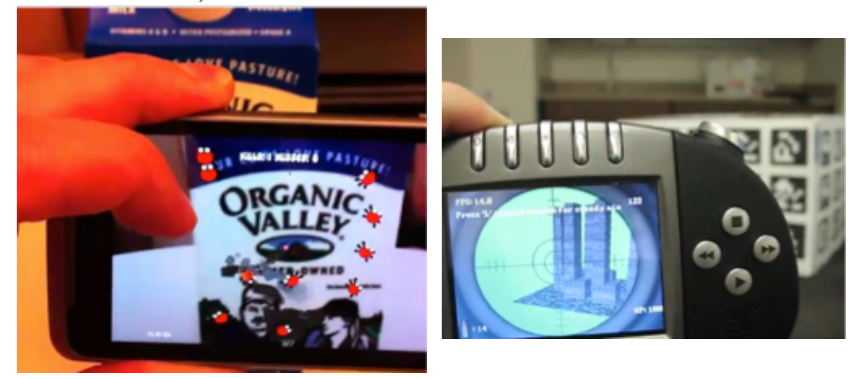

Figure 1. (Left) Bug Juice uses the metaphor of a magnifier. It renders a hot spot in the center of the screen, which makes a sizzling sound and leaves burning trails behind. (Right) Joe Warpin renders the sniper lens as a visual cue of the device metaphor of sniper gun.

In another example is a shooting game called Joe Warpin, the metaphor of sniper in a helicopter is used. The sniper reticule rendered on the screen suggests the actions of aiming and shooting. Helicopter noise and the unsteady movement of the reticule on the screen add realism. Holding a button (to hold their breath for a short time) steadies the target.

Using the pre-pattern: When leveraging metaphors, designers must be aware that they do not always transfer existing knowledge perfectly to a new medium. Metaphors can be potentially impeding if too much discrepancy exists between them.

For example, we have seen another category of metaphors, "paddles", in HAR games [45]. However, when applying "paddle" metaphors, a designer needs to keep in mind that the display is attached to the device and moves along with it. Moving the display causes problems in games that require the player to receive visual feedback. In this case, designers need to either compensate with other sensory modalities or create game mechanics that encourage stable hand-eye position.

\subsection{Control mapping}

Definition: A HAR interface, as a game controller, supports a set of physical actions that can be intuitively mapped to the actions in the game. A designer needs to make conscious decisions about selecting the control actions and mapping them in the game.

Description: Handheld devices provide a variety of control mechanics, such as touch screens and accelerometers. HAR technology supports a set of interaction mechanisms that are unique, due to its physical presence in the space and the firstperson view "through" the device. Through HAR interfaces, users can perform actions via three mechanisms: 1) projection from screen to space, 2) physical object manipulation, and 3) device manipulation. These actions need to be intuitively mapped to the actions in the game.

We break down the possible mappings as follows:

1). Projection from screen to space. A common approach is the ray casting mechanic that has been widely co-opted from other $3 \mathrm{D}$ games (e.g., the two games shown in Figure 1). In this case, a ray is cast from the device screen to the virtual world, either originating from the center of the screen or from a point touched by the user. This category of actions relies on a player's familiarity with the action of pointing and aiming, which is mapped to the actions of focus and selection in the digital game world.

2). Physical object manipulation. A player can move, rotate, and tilt tracked objects in order to affect the virtual objects in an analogous way (see Figure 3 (Right)).

3 ). Device manipulation. A player may pan, zoom (Figure 2), and shake the handheld device (e.g., the "earthquake attack" in InviZimals [47] is launched by shaking the device). Movements of the device can affect the game state either by changing the movement of the characters or changing the environment.
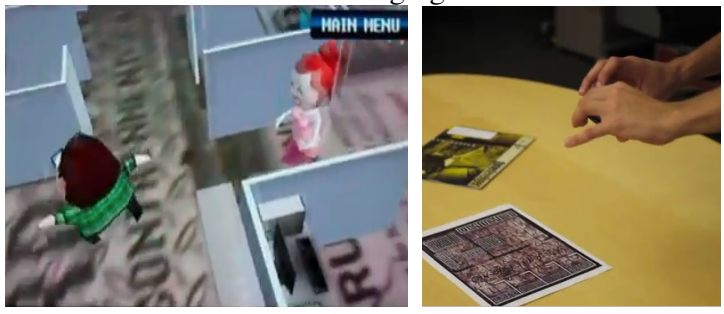

Figure 2. In Nerdherder, the device repels the nerds (Left) A nerd runs from the device. (Right) A player's physical action of moving the device closer to the Nerds to scare them away.

This pattern is not just about the game control mechanics, but about the design trade-offs as well. Often different physical actions can be mapped to the same controls, resulting in games that feel and play very differently. Two gadget based puzzle games, Inch High Stunt Guy ${ }^{2}$ and Candy Wars, are both controlled by arranging objects. The former projects the touch screen events to the digital objects on the tabletop, while the latter maps the position and orientation of physical game pieces to digital gadgets (Figure 3). Inch High Stunt Guy is more accessible since a player

${ }^{2}$ Inch High Stunt Guy, by Defiant Development (defiantdev.com), 2011 
only needs to print one trackable sheet, while Candy Wars creates a greater sense of control with tangible game control mechanics.

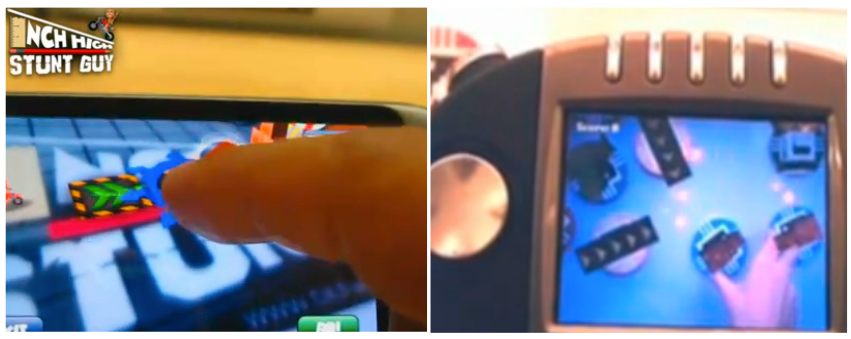

Figure 3. Similar mechanic and different controls in Inch High Stunt Guy (left) (Courtesy of Morgan Jaffit) and Candy Wars (right)

Examples: The examples are included in the description.

Using the pre-pattern: To create an intuitive and enjoyable control mapping, designers need to consider which action gives the player the maximum sense of control and satisfaction, taking into account the players cognitive load [24], the characteristics of the technology and the context in which the game is expected to be played. One common pitfall is to add "novel" game controls that seem compelling but are either unnecessarily complicated, or that do not work well in real use.

When we designed Art of Defense (See Figure 4), a tower defense game, we experimented with placing units on the tiles by either placing a token on the tile (Figure 4. middle), or drawing a shape in the middle (Figure 4. right). There was a trade-off between the granularity and richness of control (e.g., analyzing the quality of the drawn shape to influence the power and capabilities of the resulting unit) and the simplicity of the interaction. After play testing, we decided that the token-based actions were more intuitive because they followed conventions from board games, and that they were more robust in practice.


Figure 4. Constructing the space by maneuvering tracked objects. (Left) A tower that was built. (Middle). The tokens and markerbased tiles. (Right). Sketch-based building.

\subsection{Seamful Design}

Definition: HAR interfaces have inherent limitations because of their use of computer-vision based tracking. Designers need to explicitly design for the seams by various means: limiting the action to a range of in a natural way, helping users when tracking is lost, or continuing the game experience despite loss of tracking.

Description: In Chalmers et al.'s work, the authors brought forward the concept of "seamful design", in which designers take advantage of the physical limits and characteristics of a medium, rather than ignoring them [54]. In their game design, they actively design for the seams of variable coverage of Wi-Fi access points, allowing player to enjoy the game as it switches between on-line and off-line mode [55]. Most HAR interfaces currently rely on vision-based tracking which will fail outside a limited range of distances, angles, device motion and lighting. These seams are mostly seen as technology failures, but we believe that the seams in HAR interfaces can be part of the game design. When developing games for the mass market, these breakages are not show-stoppers if the seams are designed for properly.

Examples: Here we present three examples of how the seams can be handled gracefully. The first example subtly reminds the player to stay within the boundary of technology limits. In the game Joe Warpin, the metaphor of a sniper in a helicopter is used to remind the players to stay certain distance away from the tracking surface. The reason for this design choice is because of the limits of the fiducial marker tracking used in the game, requiring players to see at least one complete marker in their camera view. To retain sufficient distance, the game gives feedback that blends well with the theme of the game.

The second example creates a continuous game experience even when the seams occur. In the game of Paparazzi ${ }^{3}$, a phototaking game where the player gains points by taking pictures of a celebrity, the early part of the game requires the player to look at a character walking around on the tracking surface, from different angles. During the game, the character jumps at the phone and hangs onto its edges, dangling from the phone screen. During this phase of the game, the player can completely ignore the tracking surface because the interaction is controlled entirely be the accelerometers in the device. In this case, the game designers have created a game experience with two modes of interaction, each of which is well suited to the limits of the technologies. The switch between them is handled transparently and smoothly.
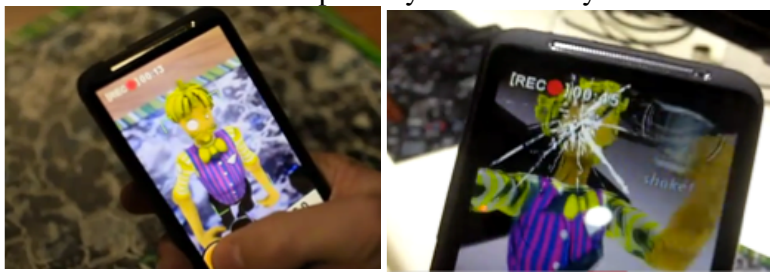

Figure 5. Paparazzi, a HAR photo-taking game. (Left). Player taking a picture while tracked; (Right) a different game state with just accelerometer input. (Courtesy of Paulius Liekis)

In some HAR games, when tracking is lost, the game gives the user some indication and reminder to help them to restore the tracking; for instance, in Nintendo 3DS AR games [6], the screen displays static. In such cases, the player is pulled out of the game experience and reminded to "repair" the technology by looking back at the marker card. In contrast, if the designer tries to have the game experience continue, the user may not realize that the technology has failed, and become frustrated. For example, through informal play testing with children, we have found that in the EyePet game for the Sony PSP [48], children did not notice the small textual messages indicating tracking was lost, and were frustrated when the pet "chose" to jump into it's bubble (Figure 6 right).


Figure 6. The explicit indication of lost tracking (in the Nintendo 3DS AR game) and the less explicit text-based reminder with the character moving in a bubble (in the PSP EyePet)

\footnotetext{
${ }^{3}$ Paparazzi, by pixel-punch (pixel-punch.com), 2011
} 
Using the pre-pattern: This pattern is used when designing around the limitations of the technology. As tracking technology and hardware improve, the limitations of technology will change. However, technology constraints will be never be completely eliminated by performance enhancements, as designers will continue to experiment with the boundaries of the technology and design for the seams.

\subsection{World Consistency}

Definition: When a player experiences a hybrid world that mixes physical and virtual realities, she naturally expects that the hybrid world will adhere to principles of the real world, such as the laws of physics. Game designers can choose to create realistic experiences by replicating properties of the real world, or they may choose to defy user expectations.

Description: By definition, HAR games create a hybrid reality through the process of registering virtual content with the physical world. Players engaging with this hybrid reality hold intuitive expectations that it will function like the real world [56]. They naturally assume that physical properties, such as gravity, collisions and spatial containment, will hold true in the hybrid environment. For utilitarian AR applications, such as maintenance and repair systems, it may be best to create a hybrid reality that closely adheres to principles of physical reality. However, games allow for unique experiences that defy player expectations. A hybrid reality may have gravity operating at different strengths, or may allow spaces to overlap. It is important to understand that players have unconscious expectations of hybrid reality based on their embodied experience of naïve physics and body awareness [4], as well as previous knowledge of games. Game designers can choose to either replicate the properties of physical reality, creating a realistic experience where players can intuitively transfer their knowledge of the real world, or may choose to defy user expectations, creating surprise and challenge for players.

Examples: The game Candy Wars relies on meeting a player's expectations of spatial relationships and naïve physics. In the game, players manipulate physical widgets on a vertical surface in order to move virtual candies from the origin to a destination. The candies are affected by gravity similarly to the way that they would if they were real, and the widgets act like physical objects such as beams and springs, applying virtual forces that alter the trajectory of the virtual candy (see Figure 3. Right). The interactions between candy and widgets replicate what a user would expect from their experience with real-world physics.

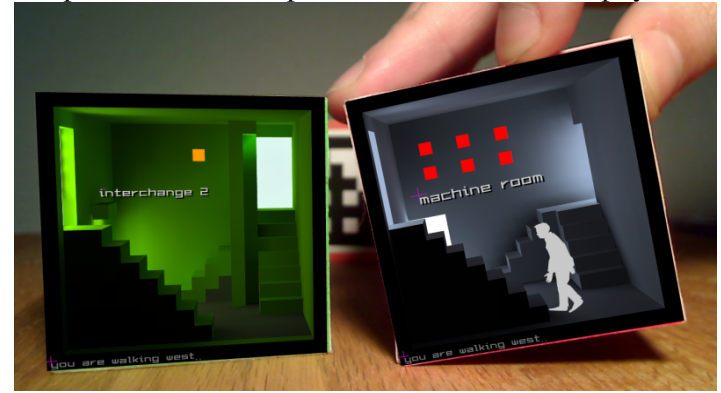

Figure 7. In LevelHead, An individual room is rendered inside each of the surface of the cubes. (Courtesy of Julian Oliver)

Another example, Levelhead $^{4}$, replicates physical reality by adhering to simplistic notions of gravity and perspective, but defies conventional understandings of space by mapping multiple volumetric spaces into a single physical volume. Each face of a physical cube corresponds to a different "room" within the cube. This mapping is not possible in physical reality since physical spaces cannot overlap; the "puzzle" aspect of the game emerges from this unconventional spatial mapping. The challenge for players is to create a mental map of the connections between the rooms inside the cube, and use tilting motions to move the game character through multiple rooms to its destination.

Using the pre-pattern: Consciously deciding to maintain predicable aspects of physical reality, or defying these in order to take advantage of possibilities inherent to virtual environments, creates different types of game. Mappings can either conform to user expectations or defy them. This choice should be made deliberately and applied consistently throughout the game design to create a predictable game experience, or else any inconsistency should have an apparent and endogenous explanation so as to maintain immersion.

\subsection{Landmarks}

Definition: Players orient and navigate through a game space by using landmarks as points of reference. Incorporating physicaldigital landmarks helps players to navigate in the hybrid space, share an external reference, and supports a spectator experience.

Description: Cognitively, for a person to navigate in a space they orient themselves through a frame of reference relative to themselves or to an external system [57]. Landmarks are readily identifiable objects that serve as external reference points [58], such as "the church," "intersection," "the white building," etc. While navigating real-world spaces, people use physical landmarks to build cognitive maps, which aid in orientation and navigation. Similarly, landmarks have been adopted in virtualreality spaces to support user's navigation in the 3D virtual space [59]. For HAR games and applications, landmarks often function in both virtual and physical worlds, which not only helps player to navigate in the hybrid space, but also serves to help them understand the relationship between the two worlds. This connection is especially important for establishing a shared external reference system when players communicate about physical-digital objects. A landmark often carries salient features that can be referred to verbally (for example, the landmark in the ARhrrrr!! game in Figure 8 can be referred to as the fountain or city center), which provides an external reference system that does not rely on the relative position and orientation of other players.

Physical landmarks also support a spectator experience [60], where a third person can gain some insight of the game state and on-going activities by observing what landmarks players are near. This latter point is important since HAR interfaces are small, individual displays and observing a game may be difficult for a non-player.

Examples: In the zombie shooting game of ARhrrrr!!, the design of the physical game board and digital space align with each other. In the 3D digital world, the streets all point towards a central focus: the fountain in the center of the city as a portal where civilians escape from the zombies. On the physical board, the same design (but in 2D) draws a player's eye towards this center. This highly recognizable physical-digital landmark helps players cognitively connect the physical and digital worlds.

Landmarks can be in other forms. For example, in LevelHead (as shown in Figure 7), the landmarks include text-based labels and different ambient light colors for different rooms.

\footnotetext{
${ }^{4}$ LevelHead, by Julian Oliver

(http://selectparks.net/ julian/levelhead/), 2008
} 


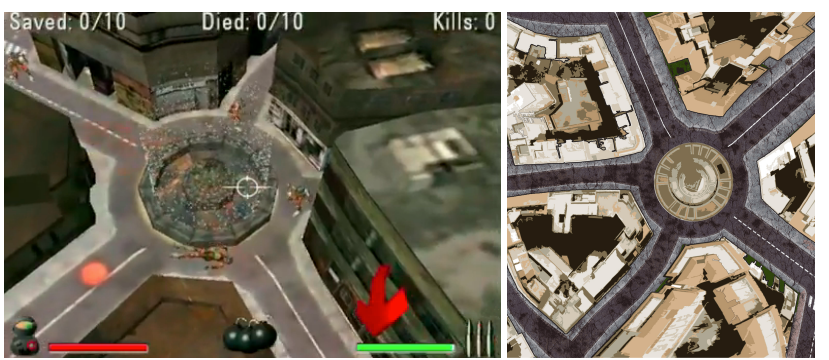

Figure 8. The matching digital and physical landmarks (e.g., the fountain in the center) that are highly recognizable in ARhrrr!!. (Left) 3D game world; (Right) 2D trackable game board.

Using the pre-pattern: Previously, with the pre-defined blackand-white fiducial markers (e.g., AR toolkit markers), it was hard to create landmarks that carry meaning in the physical world (Figure 9. left). With the development of more powerful naturalfeature-based tracking algorithms that support using images as landmarks, the trackable targets may carry meanings on their own (Figure 9. right).
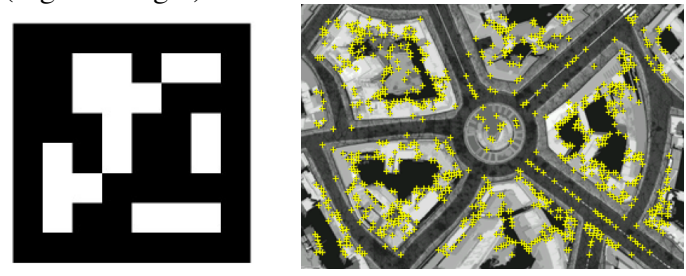

Figure 9. The comparison between a fiducial marker (AR toolkit simple marker) and a natural-feature-tracking based image target with feature points analyzed

\subsection{Living Creatures}

Definition: To create an illusion that a digital game creature lives in the physical space, game characters are designed to react to physical events, including sound, player's physical movement and hand gestures.

Description: Creating artificial life has been a longstanding dream of game designers, toy builders, roboticists, and artificial intelligence researchers. By adopting social psychology, Isbister discussed how to design the appearance and behaviors of game characters to create a socially engaging experience and evoke genuine emotions [61].

There have been physical toys that are designed to become "alive" in the digital world. For example, Webkinz [62] are stuffed animals that become playable characters online. However, this experience is made of two parts that are only thinly connected; the digital and physical aspects are divided by the boundary of the computer. A HAR interface solves this problem by blending the physical and digital experience into one. With AR, the phenomenological boundary between physical and digital worlds is blurred, and it generates a sense that the virtual objects exist in the real world, and that the player is part of the game.

Examples: With a HAR interface, a player can visually situate [12] the digital characters in a physical world. As shown in the EyePet trailer (Figure 10 (left)), a player brings his EyePet outdoors and puts it on the swing. The camera detects the motion and the EyePet creature reacts as if it is curious about the movement. A game character can exhibit the behaviors of a living being, such as playing, eating, dressing up, sleeping and even dreaming, as if they live on the trackable surface. Moreover, the system can detect physical events, such as a player moving his hand or placing objects in the character's environment, and the game characters can be designed to respond to these events to create a sense of inhabiting the physical space. For example, in the Figure 10 (right), a boy blows into the microphone to trigger the "rolling" action of the EyePet creature.
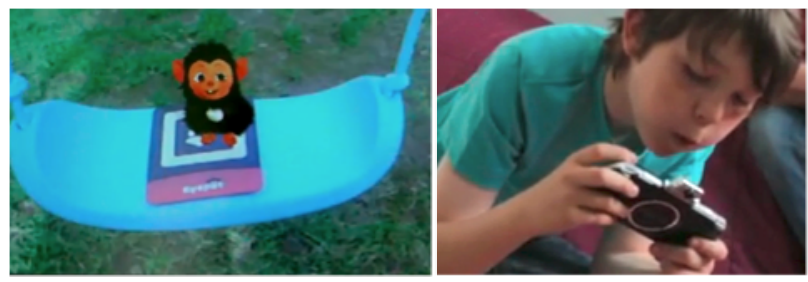

Figure 10. (Left) Situating the EyePet in a real world object swing; (Right) Incorporating real world events (e.g., blowing wind to the microphone) to affect the actions of the characters

Using the pre-pattern: With the development of the tracking technology, the characters can also have their physical forms that is closer to a living creature and still can be tracked by the handheld device. For example, in the conceptual movie about a future AR storytelling application $\operatorname{Suwappu}^{5}$ (Figure 11), the character design includes material and form factor design, and the graphics design needs to be trackable. The creature is meant to be cute and playful on its own, with or without using HAR interface.

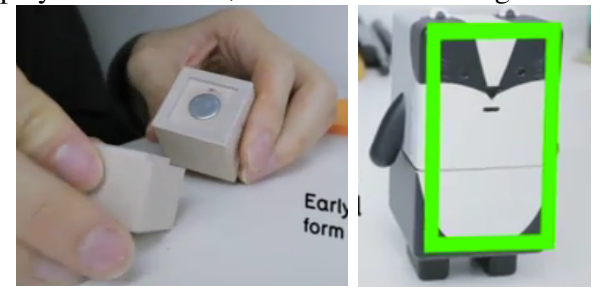

Figure 11. The material design and graphics design of the game characters in Suwappu. (Courtesy of Beeker Northam)

\subsection{Personal presence}

Definition: A player can enhance their personal presence, the sense of being in a digital game, by leveraging the HAR interface in different ways.

Description: Nitsche defines the sense of presence as "the extent to which and reasons why you feel like you are in a virtual world" (p. 205) [63]. In the conventions of existing digital games, a player's presence in a digital game can occur in very different ways, oftentimes depending on the game genre: in first person shooter games, a player adopts a first person view to convey the feeling of being in the game space; in role-playing games, avatars are typically controlled from a $3^{\text {rd }}$-person perspective; and in strategy games, the player is not directly represented in the game but is instead similar to a god, where he is able to directly manipulate the game environment. Games built for the HAR interface can support these different mechanics, and may provide a stronger sense of presence compared to traditional interfaces.

Examples: The Nintendo $3 D S$ shooting game is an example of a first-person-shooter HAR game. In this game, a dragon lives inside the marker, and it always tries to face the camera and bite the player. When the dragon attacks, the player can dodge the attack by moving the device out of the dragon's path (see Figure 12. left). Although the player does not have a visual representation in the game, the dragon senses and reacts to the movement of the player's device, reinforcing the effect that the player is present in the hybrid game space. The direct coupling between a user's

\footnotetext{
${ }^{5}$ Suwappu, by Dentsu London (dentsulondon.com)
} 
physical movements and the movement of their virtual persona may lead to stronger sense of presence.

A second kind of presence is to control an avatar so that it can move on behalf of the player. For example, in Rock'em Sock'em Robots prototype, a player controls their avatar via a touch screen "joystick " and "buttons" (Figure 12. middle). In such HAR games, the perspective of the player may not always align with that of the avatar, since the camera control is detached from the movement of the avatar. Thus, the game needs to be designed so that a player can intuitively control their avatar, and remember where it is (if they look away).


Figure 12. Player's personal presence in different games. (Left) Nintendo 3DS AR game, with the dragon always facing towards and attacking the player; (Middle) Player controlling their avatar in Rock'em Sock'em; (Right). In PuppyPlus, the player acts like "God," bringing food to the puppy on the island

Games do not necessarily require players to have a personal representation in the game. With a HAR interface, a player can participate in the game by changing the environment directly. For example, in PuppyPlus (Figure 12. right), the player's task is to provide food to a hungry puppy living on an island. The player interacts with the game by moving boats of food close to the puppy, causing the puppy to react in response. In such games, the virtual characters do not react to the player's position, but a player may change the digital world by manipulating physical objects, which may strengthen a player's presence in the game world.

Using the pre-pattern: In traditional digital games, there are conventions about how a player is represented in the game. HAR interfaces impose new meanings and usage on these conventions. With HAR, first-person presence is deepened as the physical movement of the player directly influences the game state. When the player controls an avatar in a third-person view, game designers need to ensure that the player can track the avatar and appropriately control it, since the player's view may be disconnected from their avatar. With the third-person view, a player may also directly interact with the game world using trackable game pieces or through the handheld device. Depending on the genre and theme of the game, a designer can make choices among these implementations of personal presence that are enhanced by HAR interface.

\subsection{Body constraints}

Definition: Design HAR games that take the relative position among players into account, so that when a player moves, they change the options for another player.

Description: A player's action can provide constraints to another player's subsequent moves; this strategy has been widely applied to games like Chess. A good example of leveraging bodily constraints is the board/party game Twister, in which a player occupies physical space with their body, which in turn provides possibilities and constraints for the other players' next moves.

With a HAR interface, the body position of a player can influence the performance in the game. As players move in the space, they may occupy positions that are strategically important, and the next player's moves need to be changed accordingly.

This pattern provides motivations for players to raise their awareness of, and strategically leverage, their physical movement and body position during game play. Moreover, this pattern is related to the social conventions of body distance and personal space. A social game may challenge and experiment with these social conventions to create novel social experience.

Examples: In BragFish, some players reported physically occupying a position where many fish appeared. Since it is easier to catch the fish when getting a close-up top-down view, holding this position reduces the ability of other players to fish in the same area. The physical "conflict" created many instances of shared enjoyment in our play tests (Figure 13. left).

In other games, designers try to avoid physical interference. For example, in the game Domino Knockdown (Figure 13. Right), the goal was to avoid body collisions between players. The researchers created an algorithm to shift the digital world back when players move closer to each other physically [65]. The choice between leveraging and avoiding body constraints is based on the goal of the game design.

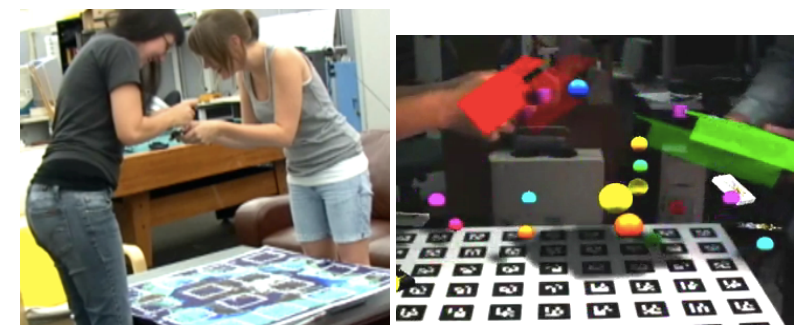

Figure 13. Two approaches to the pre-pattern of "body constraint": (Left) In BragFish, players compete to occupy certain physical location as part of the gameplay. (Right). In Domino Knockdown, the game shifts the digital world when players move closer (Courtesy of Steven Feiner and Ohan Oda)

Using the pre-pattern: This pre-pattern needs to be considered in both competitive and cooperative games. In cooperative games, body constraints can be a negative factor if they are not carefully designed for. When players cooperate in the game Art of Defense, players sometimes bump into each other, which is frustrating. To incorporate body constraints in the game, they must be tied to meaningful actions that change game state. The key is to consider the physical space as a limited resource among all the players.

Also, when applying this pre-pattern, player relationships and social norms need to be considered so that the body constraints do not feel intrusive and awkward in social games.

\subsection{Hidden Information}

Definition: In a HAR game, most of the information is hidden on the private display. But such information can be given away with body movements or during communication. Players leverage their bodily co-presence and social skills to hide and reveal part of the information, creating suspense and tension.

Description: When playing co-located non-digital games like Mafia or Poker, a player is leveraging social skills to guess some hidden information. Player's social skills are critical for such kind of game play. Sociologist found that people consciously give the impression that they want others to have, but unconsciously give away the impressions that are less controllable but more trustworthy [64]. Human beings are naturally equipped with rich skills of discerning subtle social cues for communication. 
A HAR interface implies individual displays and private information. However, players can give away information when moving themselves/the device, or by communicating with the other players. This loose connection between the information that is hidden on devices, and the information that can be given away, can foster emergent social play and enhance suspense and tension.

Examples: We evaluated the game BragFish, a competitive fishing game where the fish can only be seen around the boat that a player controls. In this game, a player can also steal the other player's fish by ramming their boats. We found players infer the other player's state and guess their next action by body posture, movement and sound cues. For example, one player told us that his strategy is to sneak up and steal. When he noticed that the other player was holding the device steady and close to the tracking surface, he inferred that they were fishing. Once the sound confirmation of the other player catching the fish occurred, he rammed them immediately and stole the fish [7]. This example illustrates how hidden and the indirect ways it might be revealed can generate emergent play strategies.

Using the pre-pattern: HAR interface can leverage players' body movement, object manoeuvring, sound, and the of verbal communication for interaction. A designer can loosely connect such behaviors to the game state so that a player can actively try to capture others' signals or give misleading signals. To use the pattern effectively, the key is to avoid an exact one-to-one mapping between an observable behavior and the game state. The fun of this pattern is creating suspense; a player may try various actions, such as pretending, bluffing, and guessing, which create interesting emergent play.

\section{Discussion AND FUtURE WORK}

The patterns presented here are derived from HAR games. While other AR technologies have different affordances and constraints, and will therefore have their own specific design patterns, we hope some of these patterns will transfer to other configurations of AR technology, such as projection based AR or head mounted AR. Similarly, while our focus has been on HAR games, a number of the lessons we have learned apply to non-game contexts. For example, the control mapping pattern speaks to the broader issues of interaction and control of any HAR system. Similarly, the cognitive and perceptual principles underlying the landmarks pattern are relevant to understanding the relationships between the physical and virtual worlds in any HAR system.

We also recognize that the approach we have taken here is only one way of understanding AR design, and as such, it emphasizes the embodied interaction aspects of HAR games, while overlooking others such as the narrative or content design. However, design patterns are theoretically neutral, and we make no claim that these nine patterns are comprehensive; while we focus on design patterns for embodied interaction, other patterns that emphasize character relationships, or mediation of the physical world, are also possible. One of the strengths of pattern languages is that sets of patterns that stress different aspects of AR experiences can be contained within the same language and mixed together to create compelling AR experiences.

Our hope is that this paper serves to begin a long-term discussion of design patterns for AR. The evolution of pattern languages is a social process, which is critically dependent on the involvement of the community in using and discussing existing patterns, and updating and creating patterns and pattern language that suit to their new situations. This work can serve as a foundation to form a pattern language for designing AR games for game design and AR communities. In the near future, we hope to create an "AR Design Patterns" wiki that includes these design patterns, plus others that did not make it into this paper.

\section{CONCLUSION}

In this paper, we present 9 design pre-patterns based on our experience of designing and teaching HAR games, and analyzing other existing commercial and academic examples. Specifically, these pre-patterns are: device metaphor, control mapping, design for the seams, world consistency, landmarks, personal presence, living creatures, hiding /revealing information, and body constraint. The pre-patterns leverage skills of the players, and can be used to inform the design of gameplay experiences that take advantage of embodied interaction enabled by HAR interface.

In the end, the game design and research communities will determine if these patterns are useful, through application (or not) of them to their design practices. Our hope is that, by synthesizing lessons and observations from a large number of HAR games, we provide a solid foundation for thinking about designing fun and compelling HAR games.

\section{ACKNOWLEDGEMENT}

We are grateful to Qualcomm for funding this research, and to all the students in the Qualcomm AR Game Studio at Georgia Tech.

\section{REFERENCES}

[1] Nintendo, "Wii at Nintendo," 2006.

[2] Microsoft, "Kinect," 2010.

[3] R. Azuma, Y. Baillot, R. Behringer, S. Feiner, S. Julier, and B. MacIntyre, "Recent advances in augmented reality," IEEE Computer Graphics and Applications, vol. 21, 2001, pp. 34-47.

[4] R.J.K. Jacob, A. Girouard, L.M. Hirshfield, M.S. Horn, O. Shaer, E.T. Solovey, and J. Zigelbaum, "Reality-based interaction: a framework for post-WIMP interfaces," Proceedings of CHI, ACM, 2008, p. 201-210.

[5] K. Kroeker, "Mainstreaming augmented reality," Communications of the ACM, vol. 5, no. 7, 2010, p19-21.

[6] Nintendo, "Nintendo 3DS," 2011.

[7] Y. Xu, M. Gandy, S. Deen, B. Schrank, K. Spreen, M. Gorbsky, T. White, E. Barba, I. Radu, J. Bolter, B. MacIntyre, "BragFish: exploring physical and social interaction in co-located handheld augmented reality games," Proceedings of ACE, ACM, 2008, p. 276-283.

[8] D.N.T. Huynh, K. Raveendran, Y. Xu, K. Spreen, and B. MacIntyre, "Art of defense: a collaborative handheld augmented reality board game," Proceedings of SIGGRAPH Symposium on Video Games, ACM, 2009, p.135-142.

[9] B. MacIntyre, K. Spreen, D. Cochard, T. Tseng, R. Summers, and K. Baskett, "ARhrrrr!! A First-Person, Fast-Action TableTop Augmented Reality Game," IEEE Virtual Reality Video Program, 2010.

[10] E. Barba, Y. Xu, B. Maclntyre, and T. Tseng, "Lessons from a class on handheld augmented reality game design," Proceedings of FDG, ACM, 2009, pp. 2-9.

[11] M. Billinghurst and H. Kato, "Collaborative augmented reality," Communications of the ACM, vol. 45, 2002, pp. 64-70.

[12] S. White, "Interaction and Presentation Techniques for Situated Visualization," PhD Diss., Columbia University, 2009.

[13] J.W. Chastine, "On inter-referential awareness in collaborative augmented reality," PhD Diss, Georgia State Univeristy, 2007.

[14] A. Dünser, R. Grasset, and M. Billinghurst, "A survey of evaluation techniques used in augmented reality studies," $A C M$ SIGGRAPH ASIA 2008 courses, ACM, 2008, pp. 1-27.

[15] T. Nilsen and J. Looser, "Tankwar-Tabletop war gaming in augmented reality," 2nd International Workshop on Pervasive Gaming Applications, 2005.

[16] M. Dunleavy, C. Dede, and R. Mitchell, "Affordances and Limitations of Immersive Participatory Augmented Reality 
Simulations for Teaching and Learning," Journal of Science Education and Technology, vol. 18, Sep. 2008, pp. 7-22.

[17] A. Morrison, A. Oulasvirta, P. Peltonen, S. Lemmela, G. Jacucci, G. Reitmayr, J. Näsänen, A. Juustila, "Like bees around the hive: a comparative study of a mobile augmented reality map," Proceedings of CHI, ACM, 2009, pp 1889-1898.

[18] H. Kaufmann and D. Schmalstieg, "Mathematics and geometry education with collaborative augmented reality," Computers \& Graphics, vol. 27, 2003, pp. 339-345.

[19] C. Alexander, S. Ishikawa, M. Silverstein, "A pattern language: towns, buildings, construction," Oxford University Press, 1977.

[20] E. Gamma and R. Helm, "Design patterns: elements of reusable object-oriented software," Addison-Wesley Professional, 1995.

[21] A. Dearden and J. Finlay, "Pattern Languages in HCI: A critical review," Human-Computer Interaction, vol. 21, 2006, p.49-102.

[22] E. Chung, J. Hong, J. Lin, M. Prabaker, J. Landay, and A. Liu, "Development and evaluation of emerging design patterns for ubiquitous computing," Proceedings of DIS, ACM, 2004, p. 233-242.

[23] T.S. Saponas, M.K. Prabaker, G.D. Abowd, and J.A. Landay, "The impact of pre-patterns on the design of digital home applications," Proceedings of DIS, ACM, 2006, p.189-198.

[24] J. Schell, "The Art of Game Design: A book of lenses," Morgan Kaufmann, 2008.

[25] S. Björk and J. Holopainen, "Patterns in game design", Cengage Learning, 2005.

[26] G. Lakoff and M. Johnson, "Metaphors we live by," Pine Forge Express, 1980.

[27] P. Dourish, "Where the action is," MIT press Cambridge, 2004.

[28] J.O. Borchers, "A pattern approach to interaction design," AI \& Soc, vol. 15, 2001, pp. 359-376.

[29] M. Van Welie and G.C. Van der Veer, "Pattern languages in interaction design: Structure and organization," Proceedings of INTERACT, Chapman and Hall, 2003, p.1-5.

[30] J.O. Borchers, "A pattern approach to interaction design," Cognition, Communication and Interaction, Springer, 2008, p. 114-131.

[31] S. Seif El-Nasr, Magy and Aghabeigi, Bardia and Milam, David and Erfani, Mona and Lameman, Beth and Maygoli, Hamid and Mah, "Understanding and evaluating cooperative games," Proceedings of CHI, ACM, 2010, pp. 253-262.

[32] D. Milam and M.S. El Nasr, "Design patterns to guide player movement in 3D games," Proceedings of SIGGRAPH Symposium on Video Games, ACM, 2010, pp. 37-42.

[33] A. MacWilliams, T. Reicher, G. Klinker, and B. Bruegge, "Design patterns for augmented reality systems," International Workshop Exploring the Design and Engineering of Mixed Reality Systems-MIXER 2004.

[34] T.W. Malone, "Heuristics for designing enjoyable user interfaces: Lessons from computer games," proceedings of $\mathrm{CHI}$, ACM, 1982, p. 63-68.

[35] R. Pausch and R. Gold, "What HCI designers can learn from video game designers," Proceedings of CHI, ACM, 1994, p. 177-178

[36] D.B. J.turner, Truna aka, Workshop on HCI and Game interfaces: A long romance, OZCHI 2010.

[37] S.E. Lindley, J.L. Couteur, and N.L. Berthouze, "Stirring up experience through movement in game play: effects on engagement and social behaviour," Proceedings of CHI, ACM, 2008, p. 511-514.

[38] F. Mueller, S. Agamanolis, and R. Picard, "Exertion interfaces: sports over a distance for social bonding and fun," Proceedings of CHI, ACM, 2003, pp. 561-568.

[39] T. Fullerton, C. Swain, and S. Hoffman, "Game Design Workshop: Designing, prototyping, and playtesting games," Focal Press, 2004.
[40] J. Juul and M. Norton, "Easy to use and incredibly difficult: on the mythical border between interface and gameplay," Proceedings of FDG, ACM, 2009, p. 107-112.

[41] J. Chastine and Y. Zhu, "The cost of supporting references in collaborative augmented reality," Proceedings of GI, Canadian Information Processing Society, 2008, p. 275-282.

[42] C. Tan and D. Soh, "Augmented Reality Games: A Review," In Proceedings of GAMEON-ARABIA, EUROSIS, 2010.

[43] SCE Japan Studio, The Eye of Judgment ${ }^{T M}$, Sony Computer Entertainment, 2007

[44] D. Wagner, T. Pintaric, and D. Schmalstieg, "The invisible train: a collaborative handheld augmented reality demonstrator," $A C M$ SIGGRAPH Emerging technologies, ACM, 2004, p. 12.

[45] A. Henrysson, M. Billinghurst, and M. Ollila, "Face to face collaborative AR on mobile phones," Proceedings of ISMAR, IEEE, 2005, pp. 80-89.

[46] A. Mulloni, "A collaborative and location-aware application based on augmented reality for mobile devices," Master thesis, Universita` degli Studi di Udine, 2007.

[47] Novarama, Invizimals, SCEE, SCEA, 2009.

[48] SCE London Studio, EyePet (PSP version), Sony Computer Entertainment, 2010

[49] H. Korhonen and E.M.I. Koivisto, "Playability heuristics for mobile multi-player games," Proceedings of DIMEA, ACM, 2007, p. 28-35.

[50] H. Desurvire, M. Caplan, and J.A. Toth, "Using heuristics to evaluate the playability of games," CHI'04 extended abstracts, ACM, 2004, p. 1509-1512.

[51] S. Bjork, J. Falk, R. Hansson, and P. Ljungstrand, "Pirates! using the physical world as a game board," in Human-computer interaction, IOS Press, 2001, p. 423.

[52] R. Gibbs, "Metaphor is grounded in embodied experience," Journal of Pragmatics, Elsevier, vol.26, no.7 2004, p1189-1210.

[53] J. Juul and M. Norton, "Easy to use and incredibly difficult: on the mythical border between interface and gameplay," Proceedings of FDG, ACM, 2009, pp. 107-112.

[54] M. Chalmers, M. Bell, and B. Brown, "Gaming on the edge: using seams in ubicomp games," Proceedings of ACE, ACM, 2005, p. 306-309.

[55] L. Barkhuus, M. Chalmers, and P. Tennent, "Picking pockets on the lawn: the development of tactics and strategies in a mobile game," Proccedings of UbiComp, ACM, 2005, p. 358-374.

[56] E. Hornecker and A. Dünser, "Of Pages and Paddles: Children's Expectations and Mistaken Interactions with Physical-Digital Tools," Interacting with Computers, vol. 21, 2009, pp. 95-107.

[57] G. Committeri and G. Galati, "Reference frames for spatial cognition: Different brain areas are involved in viewer-, object-, and landmark-centered judgments about object location," Journal of Cognitive Neuroscience, 2004, Vol. 16, No. 9, Pages 1517-1535.

[58] K. Lynch, "The image of the city," the MIT press, 1960.

[59] N. Vinson, "Design guidelines for landmarks to support navigation in virtual environments," Proceedings of CHI, ACM, 1999 , p. 278-285.

[60] S. Reeves and S. Benford, "Designing the spectator experience," Proceedings of CHI, ACM, 2005, p741-750.

[61] K. Isbister, "Better game characters by design: a psychological approach", Elsevier, 2006.

[62] Ganz, Webkinz, 2005.

[63] M. Nitsche, "Video game spaces: image, play, and structure in 3D worlds," The MIT Press, 2009.

[64] E. Goffman, "The presentation of self in everyday life," 1978.

[65] O. Oda and S. Feiner, "Interference avoidance in multi-user hand-held augmented reality," Proceedings of ISMAR, IEEE, 2009, pp. $13-22$. 